\title{
Sleep Deprivation and Plasma Biomarkers for Alzheimer's Disease
}

Authors: Martin Olsson, MD 1,2,3,4* Johan Ärlig, MD'1,2,3; Jan Hedner, MD, PhD ${ }^{3,4}$; Kaj Blennow, MD, PhD ${ }^{1,2}$; Henrik Zetterberg, MD, $\mathrm{PhD}^{1,2,5,6}$.

${ }^{*}$ Corresponding author: Martin Olsson, MD, Sahlgrenska University Hospital

Contact information:

E-mail: martin.v.olsson@vgregion.se

Address and Postal code: Neurochemistry, House V, Sahlgrenska University Hospital, SE-431 80, Mölndal

Telephone: (0046) 733161657, Fax: (0046) 0313432426

\section{Authors' affiliations:}

${ }^{1}$ Clinical Neurochemistry Laboratory, Sahlgrenska University Hospital, Mölndal, Sweden

${ }^{2}$ Institute of Neuroscience and Physiology, Department of Psychiatry and Neurochemistry, University of Gothenburg, Sahlgrenska University Hospital, Mölndal, Sweden

${ }^{3}$ Center for Sleep and Vigilance Disorders, Department of Internal medicine, University of Gothenburg, Sahlgrenska University Hospital, Gothenburg, Sweden

${ }^{4}$ Sleep Laboratory, Pulmonary Medicine, Sahlgrenska University Hospital, Gothenburg, Sweden ${ }^{5}$ Department of Molecular Neuroscience, UCL Institute of Neurology, Queen Square, London, UK

${ }^{6}$ UK Dementia Research Institute at UCL, London, UK

\section{Authors' contributions:}

Zetterberg and Olsson had full access to the data in the study and take full responsibility of the accuracy of data analysis and the integrity of the data.

Study concept and design: Olsson, Ärlig, Hedner, Blennow, Zetterberg Obtained funding: Hedner, Blennow, Zetterberg.

Study supervision: Hedner, Blennow, Zetterberg.

Data acquisition, analysis and/or interpretation: Ärlig, Olsson, Hedner, Blennow, Zetterberg.

Statistical analysis: Olsson, Zetterberg.

Drafting of the manuscript: Olsson, Zetterberg.

Manuscript revision for critical intellectual content: All authors 


\section{To the editor}

Studies suggest that sleep is important for brain-to-blood clearance of several Alzheimer's disease (AD)associated proteins, potentially through the glymphatic system (1). Human data have shown a correlation between total sleep deprivation and an increase of CNS-derived biomarkers in cerebrospinal fluid (2). Our group recently published data from an experiment where 13 healthy adults were subjected to partial sleep deprivation (PSD) (5 consecutive nights with 4 hours of sleep) (3). This intervention did not affect CSF biomarkers associated with $A D$. We have now performed a follow up analysis of plasma from the same experiment showing unaltered concentrations of amyloid $\beta 40$, amyloid $\beta 42$, tau and neurofilament light (Table).

The interpretation of the current findings is not obvious; a negative result and a small sample size introduces a risk of a type two error. However, our data suggests that a more "clinically relevant" sleep deprivation protocol does not produce a profound change in CNS protein dynamics, as protein transport from the interstitial space or CSF to plasma does not seem to be affected. Neither CSF nor plasma mirrors the interstitial space fluid chemistry perfectly but has a useable correlation (4).

It is important to emphasise that we have not examined CNS clearance of these AD-related proteins in older adults at increased risk of AD. Our PSG data suggest that a young and healthy brain responds to PSD so that the duration of SWS/NREM stage 3 is maintained, which in turn suggesting that SWS is the sleep phase during which the glymphatic clearance system is most active. This could protect from buildup of potentially neurotoxic proteins and metabolites during the times of PSD that people so often experience during normal life. The situation could be different in the aging brain with a diminishing SWS potion of normal sleep. Further studies are needed to clarify when, or if, sleep becomes a limiting factor for protein clearance from the brain.

Keywords Alzheimer disease, amyloid beta, tau, neurofilament light, sleep deprivation.

\section{References}


1. Tarasoff-Conway JM, Carare RO, Osorio RS, Glodzik L, Butler T, Fieremans E, et al. Clearance systems in the brain-implications for Alzheimer disease. Nature reviews Neurology. 2015;11(8):457-70.

2. Ooms S, Overeem S, Besse K, Rikkert MO, Verbeek M, Claassen JA. Effect of 1 night of total sleep deprivation on cerebrospinal fluid beta-amyloid 42 in healthy middle-aged men: a randomized clinical trial. JAMA neurology. 2014;71(8):971-7.

3. Olsson M, Arlig J, Hedner J, Blennow K, Zetterberg H. Sleep deprivation and cerebrospinal fluid biomarkers for Alzheimer's disease. Sleep. 2018;41(5).

4. Brinker T, Stopa E, Morrison J, Klinge P. A new look at cerebrospinal fluid circulation. Fluids and barriers of the CNS. 2014;11:10.

5. Shokri-Kojori E, Wang GJ, Wiers CE, Demiral SB, Guo M, Kim SW, et al. beta-Amyloid accumulation in the human brain after one night of sleep deprivation. Proc Natl Acad Sci U S A. 2018;115(17):4483-8. 


\section{Plasma biomarker data}

Baseline (CS) vs PSD

Mean (SD)

Variable
Baseline (CS) Partial Sleep Deprivation

$(\mathrm{N}=13)$

$272.4(28.2)$

14.3(2.2)

2.8(1.1)

6.1(5.7)
$(\mathrm{N}=13)$

276.3(26.2)

$14.5(2.4)$

3.0(1.3)

$5.5(4.1)$
$P$ Value

Prolonged PSD

$(\mathrm{N}=4)$

$275.8(37.0)$

0.70

14.8(2.1)

Tau, pg/mL

$\mathrm{NfL}, \mathrm{pg} / \mathrm{mL}$

0.28

3.0(0.6)

0.42

7.5(4.2)

Abbreviations: CS, controlled sleep. PSD, partial sleep deprivation. A $\beta, \beta$-amyloid. NFL, neurofilament light.

P-values represent within group (the same subjects exposed to two sleep conditions) differences for the controlled sleep period samples compared with the partial sleep deprivation samples. P-values have not been calculated for the prolonged PSD group because of the small samplesize. 